\title{
OBSERVAÇÃO DE LINHAS DE APRENDIZAGEM COLABORATIVAS ${ }^{1}$
}

\author{
OBSERVATION OF COLLABORATIVE LEARNING LINES ${ }^{1}$
}

\author{
Jairit Garavit ${ }^{2}$ \\ https://orcid.org/0000-0003-1976-9891
}

1. ¿Este produto é o resultado do trabalho árduo de uma de suas Linhas de Pesquisa do projeto "Como deve pesquisar em Educação virtual e a distância?" do ano de 2021, realizada com Membros de forma interdisciplinar e interinstitucional (Professores - Alunos) da Pesquisa Seedbed: Sistemas Integrados de Gestão "Integrados" Anexados ao GrupLAC: SIGCIENTY da Universidade Nacional Aberta e a Distância UNAD.

2. Engenheiro Industrial, Especialista em Educação Superior e à Distância, Mestre em Sistemas Integrados de Gestão HSEQ-RSC, Revisor de Pares da Elsevier, Revisor de Pares da Publons Academy e Advisor \& Peer Rewiever de Mendeley, Editor de Currículo em Revistas Acadêmicas - Minciencias. Líder do "Integradoss" Research Seedbed e nomeado Research Professor da Escola de Ciências Básicas, Tecnologias e Engenharia - ECBTI Caribbean Zone, da National Open and Distance University - UNAD. Email: Jairit.Garavit@unad.edu.co

Projeto de investigação: ¿Cómo investigar a Educação virtual e a distância? 
Resumo: Em um alto nível, a colaboração é considerada uma habilidade suave, uma parte fundamental de um bom aluno, e saber como colaborar em equipe tem um grande efeito na produtividade. No entanto, quando uma organização prioriza isso, a colaboração pode ser muito maior do que isso. Torna-se um sistema operacional que conecta recursos em uma estrutura educacional diversificada para melhorar os resultados. O principal objetivo do sistema educacional é criar uma mudança de comportamento nos indivíduos na direção desejada. $\mathrm{O}$ conceito de aprendizagem passa a ter um papel fundamental. A maneira pela qual os indivíduos são assimilados no curso real tem sido um centro de investigação e investigação. As hipóteses, operações, abordagens, guia, modos, compêndios, metodologias e processos de ensino, eles aproveitaram seu espaço na proposição e na experiência para apresentar argumentos a essa questão. O treinamento autônomo e colaborativo é um deles. Visto que, no campo de estudo, as diferenças entre o grupo do método, as características críticas do método e os sites do método, são causas de estudo aprofundado, na era das novas tecnologias de informação.

Palavras chave: Aprendizagem colaborativa, Responsabilidade pessoal, Característica crítica, Procedimentos educacionais.

Abstract: At a high level, collaboration is considered a soft skill, a fundamental piece of a good learner, and knowing how to collaborate as a team has a huge effect on productivity. However, when an organization prioritizes it, collaboration can be much greater than this. It becomes an operating system that connects resources in a diverse educational structure to improve results. The main objective of the educational system is to create a change of behavior in individuals in the desired direction. The concept of learning enters a fundamental role. The manner in which individuals assimilate in the actual course has been a center of inquiry and investigation. The hypotheses, operations, approaches, guides, modes, compendia, methodologies and teaching processes, have taken their space in the proposition and the experience to give arguments to this question. Autonomous and collaborative training is one of them. Since, in the field of study, the differences between the group of the method, the critical characteristics of the method and the websites of the method, are causes of indepth study, in the era of new information technologies.

Keywords: Collaborative learning, Personal responsibility, Critical characteristic, Educational procedures. 


\section{Introdução}

A fim de realizar um processo de ensino eficaz, a compreensão atual da educação tem confrontado os educadores com a responsabilidade de determinar o método de ensino que irá maximizar a aprendizagem e aplicação desse método. Porém, sabe-se que, no âmbito dos livros didáticos nas instituições de ensino, o coletivo de professores exerce a tradicional técnica de expressão baseada na participação dos alunos como ouvintes passivos. Alguns professores usam métodos de impressão que acreditam ativar os alunos (Simsek, Doymus, Kizloglu, 2005). No entanto, os alunos podem expressar livremente seus pensamentos individuais, discutir e cuidar dos outros. Eles adotam o Método de Treinamento Autônomo Colaborativo, que permite que as atividades de ensino-aprendizagem sejam mais eficazes,

\section{Método de treinamento colaborativo:}

O método de treinamento colaborativo foi nomeado e definido de várias maneiras. Embora sejam considerados diferentes como um conceito, todos eles têm o mesmo conteúdo em termos gerais. Demirel, Un (1987) e Acikgoz (1992); Ao nomear o conceito de colaboração como treinamento colaborativo, este conceito pode ser denominado como formação autônoma colaborativa para o aprendiz. O conceito de Treinamento Colaborativo em diferentes estudos; É denominado em paralelo com conceitos como: Grupo de Trabalho, Aprendizagem Colaborativa, Aprendizagem Coletiva, Comunidades de Aprendizagem, Aprendizagem por Pares, Aprendizagem Mútua, Aprendizagem Conjunta, Ambientes de Estudo, Grupo de Estudos, Ensino por Pares e Trabalho em Equipe. Em todas essas abordagens, há três tipos comuns de trabalho em grupo, incluindo grupos de aprendizagem informal, grupos de aprendizagem organizados (formais) e equipes de trabalho (Davis 1993). Embora o nome seja diferente, existem semelhanças significativas em termos de conteúdo. Nós, por outro lado, consideramos o conceito de "Treinamento Colaborativo" adequado.

O método de treinamento colaborativo é definido de diferentes maneiras por diferentes cientistas educacionais. Este treinamento colaborativo é um método de aprendizagem em que o aluno assume um papel ativo em vez de se concentrar no professor (Cooper e Mueck 1990), aumentando a motivação e retenção, ajudando os alunos a expandir um perfil positivo de si próprios e de seus colegas., Resolução de problemas e ideologia crítica.

Uma abordagem de aprendizagem em sala de aula que é usada para desenvolver força e promover habilidades sociais colaborativas, um ambiente de aprendizagem em sala de aula onde os alunos trabalham em pequenos grupos mistos (Watson 1992), uma abordagem de aprendizagem em que o sucesso $\mathrm{O}$ grupo é recompensado de maneiras diferentes, nas quais os alunos ajudam uns aos outros para aprender sobre um assunto acadêmico de acordo com um objetivo comum, formando pequenos grupos mistos no ambiente de sala de aula (Denle 
1998). como o processo de realizar a aprendizagem trabalhando em pequenos grupos e ajudando uns aos outros a aprender (Ac1kgoz 2003), é definido como um procedimento de aprendizagem baseado em alunos trabalhando juntos em pequenos grupos mistos, ajudando uns aos outros a aprender (Johnson e Johnson, 1992).

Como conceito, o treinamento colaborativo inclui metodologias de treinamento em que os alunos interagem em seu trabalho em pequenos grupos, geralmente de 3 a 5 pessoas, e a competição em grupo é recompensada de maneiras diferentes (Slavin 1988).

Levando em consideração as definições dadas acima, o ensino é um pilar fundamental da formação em que os alunos ajudam uns aos outros a aprender sobre um assunto acadêmico online com um objetivo comum, formando pequenos grupos mistos tanto na sala de aula como em outros. a confiança dos indivíduos aumenta, suas habilidades de comunicação melhoram, sua capacidade de coragem em complicações e acelera a ideologia crítica. Pode ser definida como uma abordagem de aprendizagem em que o aluno participa mais ativamente do processo de ensino (Doymus, Simsek, Bayrakceken 2004).

\section{Favores para a implementação deste método de treinamento}

A essência deste método de aprendizagem é que os alunos conversem juntos sobre um assunto e um problema e procurem soluções. Para que isso aconteça, cada aluno deve fazer a sua parte, e isso é necessário para que o método de treinamento colaborativo seja alcançado. A aprendizagem colaborativa também é um método que proporciona desenvolvimento social e mudança individual. Com esse método, aumenta o senso de responsabilidade do indivíduo e o prazer de contribuir para os outros e para o grupo (Buyukkaragoz e Civi 1997).

Pode-se dizer que um indivíduo que desenvolve senso de responsabilidade e gosta dessa situação quando contribui para os outros tem aumentado seu desenvolvimento emocional tanto na socialização quanto na situação em que se encontra. Na compreensão atual da educação, a socialização do indivíduo ocupa um lugar importante. Nesse sentido, o método de aprendizagem colaborativa também atinge o objetivo da participação das pessoas, que estão entre os objetivos da nossa educação, na sociedade como ser social de mais alto nível, para esse fim.

A eficacia do método de aprendizagem está aumentando gradualmente. O método de aprendizagem colaborativa não só aumenta o sucesso acadêmico, especialmente na aprendizagem complexa de alto nível, mas também melhora a confiança dos alunos uns nos outros, suas atitudes e interesses na área de assunto. Portanto, também oferece uma solução para o problema de aquisição de traços afetivos que foram negligenciados na educação e que são muito difíceis ou impossíveis de alcançar com outros métodos. Além da raspagem de 
traços afetivos, também inclui a teoria da motivação em sua estrutura. tem. O lugar da teoria da motivação neste método é na estrutura de propósito e recompensa que direciona os alunos para o trabalho.

Os membros do grupo, unidos em torno de seus próprios objetivos pessoais, precisam se apoiar e ajudar uns aos outros para que o grupo alcance o mais alto nível de sucesso, ou seja, o sucesso do grupo é eficaz para aumentar o desempenho dos membros do grupo, a estrutura de recompensa entre os sujeitos do grupo fortalece as relações sociais entre o grupo e aumenta o desejo de trabalhar juntos.

Uma vez que os indivíduos precisam trabalhar de forma colaborativa no método de aprendizagem cooperativa, ajudar uns aos outros torna-se o comportamento mais eficaz. Durante essas atividades colaborativas, é inevitável que tanto o assistente quanto o diretor se beneficiem como resultado de uma cooperação que foi completamente eliminada com explicações corajosas como reorganizar o problema, explicar e definir passo a passo como resolver o problema em ordem para passar seus pensamentos para outros amigos. Nesse processo, as colaborações fornecem aos alunos novas perspectivas e as desenvolvem. Eles são vistos como estabelecendo uma forte conexão entre o que aprenderam antes e novas informações, eliminam equívocos e são eficazes na eliminação de deficiências de comunicação entre seus amigos (Webb 1985). Em situações de treinamento colaborativo, é imperativo manter um propósito comum entre os alunos. Para que os grupos tenham sucesso, o método de aprendizagem cooperativa deve ser planejado, assim como os membros do grupo se ajudando e apoiando uns aos outros.

Os alunos podem alcançar o sucesso de seu próprio grupo com o sucesso de seus outros amigos no grupo. Isso também é importante para garantir que os membros do grupo façam o possível. O sucesso do grupo depende do desempenho de seus membros. Por este motivo, as pessoas do grupo devem ajudar-se mutuamente para atingir os objetivos individuais.

Os alunos podem alcançar o sucesso de seu próprio grupo com o sucesso de seus outros amigos no grupo. Isso também é importante para garantir que os membros do grupo façam o possível. Por esta razão, as pessoas do grupo devem ajudar-se mutuamente para atingir os objetivos individuais, os alunos podem alcançar o sucesso de seu próprio grupo com o sucesso de seus outros amigos no grupo. Isso também é importante para garantir que os membros do grupo façam o possível.

(Slavin 1990). As pessoas que conhecem a necessidade de atingir seus objetivos individuais são outras pessoas. Mais importante ainda, o grupo ajuda os membros bemsucedidos como um grupo, de modo que os elementos uns dos outros são encorajadores. Essa situação proporciona um reforço social, como elogiar ou incentivar o trabalho de outras pessoas relacionadas à área temática. 
Crie uma estrutura de recompensa interindividual (Johnson e Johnson 1988). No Método de Aprendizagem Colaborativa, quando os tópicos são escolhidos de forma adequada, há evidências de uma expansão de conceitos críticos nos alunos. Alunos no mesmo nível de desenvolvimento têm mais sucesso em atividades de resolução de problemas com o favor e orientação de seus colaboradores. Portanto, o método de aprendizagem ou treinamento colaborativo apóia o desenvolvimento mental das crianças (alunos). Isso ocorre porque alunos da mesma faixa etária gostam de trabalhar juntos. Os grupos aprendem a preparar apresentações orais e escritas em conjunto para a sua organização durante o seu trabalho, o seu próprio comportamento e o dos outros em grupos,

Ao mesmo tempo, o método de aprendizagem também tem um efeito no aumento da competência individual dos alunos, tomando como modelo os membros do seu próprio grupo ou outras disciplinas do grupo. Portanto, a atividade de treinamento colaborativo primeiro fornece treinamento cooperativo no contexto de relacionamentos entre indivíduos. Então, individualmente, dê forma aos eventos mentais. Além disso, os alunos aprenderão uns com os outros devido às suas discussões sobre um determinado tópico, surgirão diferenças cognitivas, serão expressos raciocínios inadequados e surgirão entendimentos de maior qualidade. Portanto, a interação dos alunos entre si em relação aos tópicos de aprendizagem guiará o aumento do sucesso do indivíduo (Slavin, 1990).

No Método de Treinamento Colaborativo, foi determinado que os membros do grupo costumam usar a tática de fazer perguntas, discutir e tomar notas, mas também foi determinado que eles motivam uns aos outros durante o exame da unidade, competência ou preparação de suas matérias (Webb et al, (1985), Chiu et al (2000). Pode-se dizer que estas atividades aumentam a permanência na aprendizagem e são eficazes na formação de aprendizagens significativas. Além disso, pode-se observar que diversos métodos de ensino na aprendizagem cooperativa Método Pode-se dizer facilmente que esses métodos são usados pelos membros do grupo sem estarem cientes disso.

O método de aprendizagem colaborativa permite que os alunos falem efetivamente em discussões em grupo, como clareza, racionalidade, vitalidade, naturalidade, expressão e estilo. Também ensina técnicas de estudo como perguntas e respostas, discussão livre, discussão em grupos pequenos e grandes, discussão em círculo, seminário, brainstorming. (Milis 1991, Schaible e Robinson 1995). Pode-se dizer que todas essas conquistas permitem que os alunos desenvolvam seus pensamentos, fomentem a criatividade, ganhem autoconfiança, se expressem e utilizem ferramentas e equipamentos. Atividades de ensino que não desenvolvem o pensamento dos alunos, apoiam sua criatividade e os levam ao aprendizado mecânico não são muito significativas em termos educacionais. 
O Método de Aprendizagem ou Treinamento Cooperativo fornece incentivo para aumentar a motivação e retenção, para ajudar os alunos a ampliar uma imagem positiva de si mesmos e de seus colegas, para cuidar de práticas destemidas e tendências críticas, e para desenvolver habilidades sociais colaborativas. O treinamento colaborativo é importante para todos os alunos. Os grupos são formados de acordo com alunos com diferentes habilidades, necessidades e estilos de aprendizagem e os alunos trabalham com esses grupos. No processo de trabalho em grupo, a expectativa e o esforço para atingir o objetivo da aula estão entre as características comuns do método de aprendizagem.

Espera-se que cada aluno do grupo interaja positivamente com outros alunos, ferramentas e ideias são compartilhadas. Além disso, os membros do grupo devem ser responsáveis por uma parte da disciplina e contribuir com o grupo em estudos relacionados ao assunto. $\mathrm{O}$ professor, por sua vez, assume o papel de organizador do ambiente e de assistente e apoio quando necessário (Mallinger 1998). Porém, não é possível se beneficiar de um trabalho em grupo em que os alunos não sejam direcionados para um objetivo e não sejam planejados.

Pode-se dizer que os alunos que trabalham de forma colaborativa usam mais métodos do que os que trabalham sozinhos. No entanto, (George 1994). Afirma-se que o processo, que inclui esta multiplicidade de métodos, é muito mais eficaz do que o método tradicional no desenvolvimento de aspectos como o interesse dos alunos pela aprendizagem, atividades interessantes, participação na aprendizagem, eficácia da aplicação, dependência de objetivos , e qualidade do ensino. Nesse método, há uma oportunidade de aplicar constantemente os métodos de fazer perguntas e ajudar uns aos outros por meio de longas discussões. A continuidade na prática garante que o aluno esteja constantemente ativo neste estudo. Foi determinado que quando os alunos estão bem motivados, o efeito do método de aprendizagem colaborativa é mais eficaz.

Quando o grupo é mencionado, vem à mente uma unidade social composta por duas ou mais pessoas que estão conectadas entre si e têm valores comuns entre si. Visto que as pessoas vivem juntas, elas naturalmente têm que trabalhar em grupos e em grupos. Portanto, o treinamento autônomo colaborativo é um dos métodos inevitáveis. A educação para o trabalho colaborativo tem sua contrapartida em suas atividades como um método de aprendizagem colaborativa. Observa-se que o método de aprendizagem cooperativa desenha um gráfico crescente entre os demais métodos de aprendizagem dos últimos anos.

Um dos motivos desse aumento; $\mathrm{O}$ fato de que o método de aprendizagem colaborativa pode ser aplicado com sucesso no ensino de todas as disciplinas e áreas disciplinares em todas as faixas etárias, todos os níveis de escolaridade.

Outro motivo é que se acredita que turmas lotadas dificultam a aplicação do método, embora estudos tenham mostrado que o método pode ser aplicado com sucesso em salas 
lotadas e pode estar entre os motivos para esse aumento. Será mais fácil garantir a participação ativa de todos os alunos em salas de aula lotadas e, quando aplicado corretamente, dará a cada aluno a oportunidade de perguntar, responder e expressar seus pensamentos.

No método de aprendizagem, dependência interpessoal, responsabilidade individual, liderança compartilhada, responsabilidade compartilhada entre os sujeitos do grupo pela aprendizagem dos outros, compreensão da importância das tarefas baseadas em uma boa relação de trabalho, orientando-os a aprender habilidades de comunicação interpessoal, observação e feedback do professor, e influência do grupo. Existem características básicas, como a autoavaliação do indivíduo (Johnson Take Meat 1984).

\section{Tipologias e detrações do método de aprendizagem}

Além de seus recursos básicos, este método também inclui vários recursos críticos.

\section{Conformidade interpessoal positiva}

A dependência positiva é baseada na necessidade de todos os membros do grupo de aprendizagem serem responsáveis pela aprendizagem dos outros membros. Existem várias estratégias para criar um vício positivo. O primeiro é material. Uma maneira fácil de fazer os alunos trabalharem juntos seria simplesmente fornecer um conjunto de materiais, um conjunto de instrumentos de laboratório, uma lista de perguntas, uma folha de respostas ou um livro de referência.

Ao recuperar materiais distribuídos de tempos em tempos, instruções ou informações podem ser repassadas aos alunos, ou um vício pode ser criado entre os alunos, mudando os tópicos daqueles que trabalharam com um conjunto de determinado material. Outra estratégia positiva para o vício é o dever de casa. As tarefas ou papéis dos alunos no grupo incluem uma meta, um jogo, um. Faz com que eles sintam a necessidade de ser interdependentes para atingir a meta. Especialmente nos alunos do grupo inferior, o grupo.

Múltiplas funções podem ser definidas para seus membros. Este determinado papel não só mantém o aluno na área disciplinar, mas também lhe dá a oportunidade de adquirir habilidades práticas, se desenvolver em alguma área especial ou colocar um talento em prática. Diferentes disciplinas acadêmicas requerem diferentes funções. Pessoas que desempenham funções diferentes sabem que, para o sucesso, todos devem cumprir sua

própria função. Para tanto, cada indivíduo do grupo está ciente da necessidade de contribuir 
com seus companheiros de grupo e da necessidade de seus companheiros de grupo contribuírem com eles mesmos.

A consciência de que o sucesso no final do trabalho vem com o sucesso do grupo reforça a ideia de que os outros amigos do grupo terão sucesso e impõe responsabilidade por seu sucesso. Essa responsabilidade assumida revela a dependência positiva entre os membros do grupo, necessária para o sucesso. Portanto, na maioria das salas de aula onde o método de treinamento colaborativo é aplicado, todos os membros de um grupo trabalham juntos para produzir e alcançar algo para seu propósito comum. Os membros do grupo devem ajudar uns aos outros a compreender todos os tópicos. Todos os membros do grupo devem ser consultados primeiro para obter a ajuda necessária, como compreender e resolver problemas.

Os alunos sabem que o professor não deve ser consultado sem discutir com os outros membros do grupo e que, se não conseguirem encontrar um acordo e uma solução entre eles, o professor deve ser consultado mais tarde. eles trabalham juntos para produzir e alcançar algo para seu propósito comum. Os membros do grupo devem ajudar uns aos outros a compreender todos os tópicos. Todos os membros do grupo devem ser consultados primeiro para obter a ajuda necessária, como compreender e resolver problemas. Os alunos sabem que o professor não deve ser consultado sem discutir com os outros membros do grupo e que, se não conseguirem encontrar um acordo e uma solução entre eles, o professor deve ser consultado mais tarde. eles trabalham juntos para produzir e alcançar algo para seu propósito comum. Os membros do grupo devem ajudar uns aos outros a compreender todos os tópicos.

\section{Compromisso individual}

Embora o sucesso dos indivíduos no método de aprendizagem tradicional seja determinado pelos valores que receberam nos testes e na redação, isso se limita a muito pouco valor na aprendizagem colaborativa. Em outras palavras, a pontuação que você obtém em exames e testes individualmente na aprendizagem colaborativa é de apenas 3\% (MEN 2020) de sua própria pontuação. As demais pontuações vêm de atividades de treinamento autônomo e colaborativo. Um dos principais objetivos dos grupos de aprendizagem no método é; Garantir que cada membro seja um indivíduo forte em termos de conhecimento acadêmico, habilidades sociais e comportamento, e também contribua para a realização de objetivos comuns da melhor maneira possível.

Para a realização dos objetivos comuns determinados, os membros do grupo devem cumprir as suas responsabilidades da melhor forma possível. Assim, o aluno assume uma segunda responsabilidade pelo sucesso do grupo, bem como por motivar e trabalhar com outros amigos, para além da sua responsabilidade individual. Portanto, os princípios de 
dependência positiva e responsabilidade pessoal são considerados componentes essenciais das salas de aula de aprendizagem colaborativa.

\section{Hábitos dentro do universo do grupo}

Normalmente, esses grupos são formados com base em suas realizações anteriores, raça, gênero e outras características decididas pelo professor. Outros traços característicos incluem coisas como idade, etnia, hobbies e interesses. Levando tudo isso em consideração, os alunos são colocados em grupos e um grupo com maior atividade de aprendizagem é formado (Cooper e Mueck 1990). Neste tipo de aprendizagem, os grupos são mantidos juntos por vários dias ou semanas para incutir o espírito de grupo, de modo que eles encontrem um nome para o seu grupo, desenvolvam emblemas do grupo, escolham as cores do grupo e até mesmo slogans do grupo. Essas formações, sem saber, dão origem ao espírito de grupo nelas.

\section{O papel do professor}

Para que esse método seja eficaz, o professor tem uma grande responsabilidade; Para estudos de treinamento autônomo e colaborativo; Para serem eficazes e eficientes, ambientes apropriados devem ser preparados para o uso de habilidades sociais, bem como habilidades de comunicação entre os indivíduos. Se os membros do grupo não se conhecem bem o suficiente, não confiam uns nos outros, não conseguem se comunicar com eficácia e não ajudam uns aos outros o suficiente, a eficiência do trabalho em grupo diminui.

Da mesma forma, a disciplina pedagógica passa a assumir o seu papel importante e integral (Professor), não só para fins de aprendizagem das disciplinas do curso, mas também para proporcionar aos alunos liderança, confiança no outro, abordagem empática aos acontecimentos, reconciliação e capacidade de comunicação eficaz. Além disso, no treinamento colaborativo, o professor determina os líderes do grupo que manterão o grupo ativo e ocupado durante o estudo e organizarão as atividades de aprendizagem.

Em vez de participar ativamente de um experimento, orientar os alunos no acesso à informação é uma das funções do professor no método de treinamento autônomo e colaborativo. Além desses, na aprendizagem, o professor orienta os alunos, facilitando e acelerando os estudos (Cooper e Mueck 1990).

\section{Habilidades sociais e de comunicação}

Os alunos são obrigados a seguir as regras de orientação fornecidas para suas habilidades sociais apropriadas e se unirem para adquirir conhecimentos e habilidades. Na maioria das aulas de aprendizagem colaborativa, os alunos aprendem a capacidade de criticar uma ideia, 
autoconfiança, empatia, confiança nos outros, bons relacionamentos. Eles aprendem sobre a necessidade de adquirir habilidades sociais, como a capacidade de se estabelecer. Outra habilidade social que recebe importância na aprendizagem colaborativa é a escuta ativa. Mesmo que se pense que esses comportamentos ou habilidades possam estar no aluno, as experiências mostram que essas características devem ser especificadas antes do início do estudo.

\section{Interação síncrona e face a face}

Este elemento significa que os sujeitos do grupo se encorajam, apoiam e ajudam uns aos outros, para que a aprendizagem ocorra de forma mais eficaz e eficiente. Os membros do grupo devem compartilhar como resolveram os problemas que encontraram, fazer um brainstorm e discutir o problema. Portanto, bons alunos servem como professores para outros membros do grupo, beneficiando a si próprios e a outros. Os alunos menos capazes recebem ajuda descritiva e corretiva dos membros do grupo. Nesse processo, o aprendizado ocorre imediatamente após o processo cooperativo e essas informações são armazenadas na memória de longo prazo. Os alunos que se sentem desconfortáveis ou relutantes em participar de grandes discussões em classe participam mais confortavelmente em pequenos grupos e tornam-se ativos.

\section{Interdependência e fusão social}

Jigsaw é chamado de estratégia complexa para criar interdependência. Essa técnica, também conhecida como técnica de fusão, transforma os membros dos grupos originais em grupos novos e de especialistas ao final do estudo, dando a oportunidade de verificar se todos os alunos do estudo cumpriram sua tarefa na área disciplinar. Especialmente para questões de serviço social, esta técnica é muito adequada e preferível. Mas uma desvantagem da técnica do quebra-cabeça; Demora mais e não é adequado para calouros. Ao final do estudo, grupos de especialistas formados por alunos que concluíram determinadas disciplinas são formados nos grupos principais formados na técnica do quebra-cabeça.

Os grupos de especialistas tratam primeiro de quaisquer irregularidades em seu trabalho e reconsideram o que aprenderão nos novos grupos de especialistas. Em seguida, eles planejam como ensinar seu tópico para outros amigos. O professor monitora constantemente o processo de grupos de especialistas em grupos de quebra-cabeça ensinando seus assuntos para seus amigos nos grupos principais e verifica se os grupos de especialistas estão totalmente informados. Por fim, os grupos de especialistas ensinam seu material para seus grupos principais. Ouvir e aprender algo com sua própria faixa etária é mais divertido e interessante para eles, além de motivá-los para essas atividades de aprendizagem. 


\section{Recompensas de grupo}

Conhecida como uma estratégia popular para cultivar o vício, as recompensas são obtidas quando o grupo atinge seu tema ou atende aos critérios. Essa estrutura de recompensa afeta positivamente os membros do grupo para entender o tópico ou atingir um objetivo comum. Ele permite que eles e os membros de seus grupos ajudem uns aos outros para melhor aprender e pesquisar suas áreas de estudo. A estrutura de premiação também cria uma atmosfera de competição. Esse ambiente aumenta a motivação dos alunos. Ao final do trabalho, a primeira turma recebe prêmios. Outros grupos que completam os critérios recebem recompensas diferentes, garantindo que eles fiquem mais dispostos a participar do próximo estudo, se todos os grupos forem bem-sucedidos no estudo, toda a turma é recompensada,

\section{Distinções de grupo colaborativo e tradicional}

\section{Grupos tradicionais}

As características críticas acima inerentes ao método de treinamento colaborativo distinguem grupos colaborativos de grupos tradicionais. Os estudos de grupo usando o método de aprendizagem cooperativa não são os mesmos que nos estudos de cluster aplicados em nossas escolas ou faculdades, o tema de cada cluster, o tema e a tarefa dos elementos do cluster. Portanto, cada aluno está interessado apenas na sua matéria. Como resultado, os alunos ainda trabalham individualmente (Topsakal 1999). Nesse contexto, os estudos realizados por Johnson e Johnson (1988) revelaram as diferenças entre os estudos de cluster aplicados até o momento e os grupos nos quais foi aplicado o método de treinamento colaborativo. Em consequência, 
Tabela 1.

Diferenças entre grupos colaborativos e tradicionais

\begin{tabular}{|c|c|}
\hline Grupos colaborativos & Grupos tradicionais \\
\hline $\begin{array}{l}\text { Os grupos deste tipo baseiam-se na interdependência } \\
\text { positiva entre os sujeitos do grupo. Os objetivos são } \\
\text { que todos os membros, exceto as próprias } \\
\text { competências dos alunos, sejam estruturados de } \\
\text { forma a obrigá-los a enfrentar as suas competências. }\end{array}$ & $\begin{array}{l}\text { No trabalho em grupo, os membros não dependem } \\
\text { uns dos outros. Não há dependência positiva entre } \\
\text { eles. }\end{array}$ \\
\hline $\begin{array}{l}\text { Existe uma responsabilidade individual clara e está } \\
\text { relacionada com o material pelo qual cada aluno será } \\
\text { avaliado e proficiente. Feedback uns aos outros sobre } \\
\text { o trabalho deles, para que os membros do grupo } \\
\text { saibam quem ajudar e quem precisa ser motivado. }\end{array}$ & $\begin{array}{l}\text { No trabalho de cluster, não há responsabilidade } \\
\text { individual para com o cluster. A pessoa é } \\
\text { responsável por si mesma. Não se responsabiliza } \\
\text { pelo grupo. }\end{array}$ \\
\hline $\begin{array}{l}\text { Nesses grupos, os membros são heterogêneos em } \\
\text { termos de habilidades e traços de personalidade. }\end{array}$ & $\begin{array}{l}\text { No trabalho de cluster, os grupos são homogêneos. } \\
\text { No trabalho de cluster, talento, habilidades sociais, } \\
\text { etc. não são levados em consideração. }\end{array}$ \\
\hline $\begin{array}{l}\text { Nesses grupos, todos os membros compartilham } \\
\text { responsabilidades de realizar atividades de liderança } \\
\text { dentro do grupo. }\end{array}$ & $\begin{array}{l}\text { Em grupos de aprendizagem tradicionais, um único } \\
\text { líder é atribuído e permanece o mesmo. Isso pode } \\
\text { impedir que outros membros do grupo adquiram } \\
\text { características de liderança. }\end{array}$ \\
\hline $\begin{array}{l}\text { Nesses grupos, os membros se sentem responsáveis } \\
\text { pelo aprendizado dos outros. }\end{array}$ & $\begin{array}{l}\text { Nesses grupos, os membros não se sentem } \\
\text { responsáveis pelo aprendizado de seus colegas. }\end{array}$ \\
\hline $\begin{array}{l}\mathrm{Na} \text { aprendizagem desse tipo, as habilidades sociais } \\
\text { são ensinadas diretamente. Isso inclui honestidade, } \\
\text { resolução de disputas de comunicação. }\end{array}$ & $\begin{array}{l}\text { Em estudos de cluster, esses valores costumam estar } \\
\text { no formato errado. }\end{array}$ \\
\hline $\begin{array}{l}\text { Nesse tipo de aprendizagem, o professor observa os } \\
\text { grupos e, em conjunto, encontra soluções para todos } \\
\text { os tipos de problemas. }\end{array}$ & $\begin{array}{l}\text { No trabalho em grupo, o professor se transforma em } \\
\text { alunos, ao invés de fazer observações. Em outras } \\
\text { palavras, o professor trabalha no lugar do aluno }\end{array}$ \\
\hline $\begin{array}{l}\text { Na aprendizagem colaborativa, o planejamento deve } \\
\text { ser abrangente, até mesmo diretrizes para o trabalho } \\
\text { em grupo devem ser fornecidas. }\end{array}$ & $\begin{array}{l}\text { Nenhuma orientação é dada no trabalho em grupo, } \\
\text { os alunos se preparam com o curso atual ou notas de } \\
\text { estudo que encontram. }\end{array}$ \\
\hline
\end{tabular}

Garavit, J. 2021.

\section{Divulgações de aprendizagem colaborativa}

Abaixo está um breve resumo de alguns dos estudos conduzidos para determinar o efeito da aprendizagem colaborativa e método de treinamento no desempenho acadêmico, atitude em relação à área de assunto e desenvolvimento de habilidades sociais e mudanças observadas nos indivíduos dentro do escopo do estudo. 


\section{Divulgações sobre aprendizagem colaborativa ou método de treinamento}

Ac1kgoz (1992a) conduziu dois estudos para determinar a eficácia do método de aprendizagem. Entre estes, foram comparados os resultados da metodologia de treinamento colaborativo sobre desempenho acadêmico, retenção de memória e aprendizagem afetiva de dois grupos de universitários com ensino tradicional. Em outro estudo de Ac1kgoz (1992b), foram examinados os efeitos da aprendizagem colaborativa, competência intergrupal e todas as atividades em sala de aula no desempenho e retenção de língua estrangeira. Ao final do estudo, concluiu-se que as atividades de aprendizagem colaborativa apresentaram resultados mais positivos na aquisição da capacidade de aplicar regras gramaticais em uma língua estrangeira e mantê-las na memória,

Keskinsiz (1993) investigou os efeitos do método de aprendizagem colaborativa e do método tradicional nas atitudes e realizações democráticas e tentou encontrar a refutação da questão de saber se os métodos aplicados na pesquisa afetam as atitudes democráticas e as realizações dos alunos. Ao final do estudo, a pesquisadora afirmou que os grupos experimentais nos quais o método de aprendizagem colaborativa foi utilizado apresentaram resultados mais positivos em termos de atitude democrática e conquista do que os grupos de controle nos quais o método tradicional foi aplicado.

Em um estudo realizado por Erkensiz e Tumkaya (1997), foi investigado o resultado da metodologia de aprendizagem colaborativa em acadêmicos e estratégias de aprendizagem e estudo. Os achados obtidos ao final do estudo revelaram uma discrepância explicativa entre o conjunto experimental e o grupo controle no nível de compreensão a favor do conjunto experimental. No entanto, não houve discordâncias reveladoras entre o experimental e o conjunto de controle em termos de outras variáveis.

Delen (1998) investigou o efeito do método de aprendizagem colaborativa no desempenho acadêmico no curso de estudos sociais da quinta série do ensino fundamental. Ao final do estudo, determinou-se que os resultados acadêmicos dos alunos do quinto ano do conjunto experimental, no qual foi utilizado o método de aprendizagem colaborativa, foram comparados com o tradicional. Concluiu-se que o método foi superior aos alunos do conjunto de controle ao qual o método foi aplicado.

Doymuş, Bayrakceken e Simsek (2003) investigaram o efeito da preparação do dever de casa com um grupo sobre o sucesso da preparação do dever de casa tradicional em um estudo que realizaram com alunos do primeiro ano de faculdade. Como resultado, foi determinado que os alunos que prepararam a lição de casa com o grupo tiveram mais sucesso do que os alunos que prepararam a lição de casa normal. 
Em um estudo de Doymus, Simsek e Bayrakceken (2004), foi investigado o efeito do método de aprendizagem colaborativa e do método de aprendizagem tradicional no desempenho acadêmico e na atitude na aula de ciências. Como derivações da investigação, concluiu-se que os alunos que aplicaram a metodologia de treinamento colaborativo tiveram mais sucesso nas atitudes acadêmicas e de curso do que aqueles que utilizaram o procedimento de treinamento tradicional.

Em um estudo de Simsek, et al. (2004) examinaram o efeito do método de aprendizagem em grupo nas atitudes democráticas dos alunos. Como resultado, constatou-se que os alunos que aplicaram o método de aprendizagem em grupo apresentaram uma melhora maior em termos de atitude democrática nos alunos que aplicaram o ensino normal.

Em um estudo de Simsek, Doymus e Kızıloglu (2005), eles investigaram o conhecimento e as habilidades adquiridas pelo método de aprendizagem tradicional com aprendizagem em grupo para alunos do ensino médio. Concluiu-se que os alunos aos quais foi aplicado o método de aprendizagem em grupo apresentaram mais aquisição nas orações de ciências e competências do que os alunos aos quais foi aplicado o método tradicional.

Johnson, et. Al. (1981) realizou importantes análises de tópicos sobre as causalidades da aprendizagem colaborativa no interesse acadêmico geral. Em todas essas análises, o método de aprendizagem colaborativa e o método tradicional foram comparados. Ao final do estudo, eles descobriram que o método de aprendizagem colaborativa foi mais eficaz em termos de desempenho acadêmico.

Treisman (1985) investigou o efeito do método de aprendizagem colaborativa em alunos negros em Berkley que entraram na faculdade para obter um certificado em matemática ou ciências. Embora o percentual de alunos negros que se formaram no programa de certificação em matemática com o método de aprendizagem colaborativa tenha sido de 44\%, o controle da taxa de graduação no grupo foi determinado em $10 \%$ e concluiu-se que o método de aprendizagem colaborativa maior sucesso acadêmico.

Frierson (1986) investigou se as candidatas a enfermeira negra usando o método de aprendizagem colaborativa tiveram mais sucesso nos exames estaduais de enfermagem do que suas colegas enfermeiras treinadas usando o método tradicional. Como resultado, concluiu-se que as candidatas a enfermeiras negras que usaram o método de aprendizagem colaborativa tiveram mais sucesso nos exames estaduais de enfermagem do que as outras enfermeiras que foram educadas com o método tradicional especificado.

Cooper e Mueck (1990) A educação universitária levou a pesquisas sobre as inadequações dos alunos e o método de aprendizagem cooperativa. Em sua pesquisa, eles mantiveram 
reuniões com grupos de quinze a vinte pessoas em uma universidade estadual da Califórnia sobre o método de aprendizagem colaborativa para realizar suas atividades em relação ao currículo atual. Eles discutiram postagens sobre o método de aprendizagem colaborativa nos últimos quatro anos e coletaram dados sobre os efeitos da aprendizagem colaborativa nas salas de aula de seus professores. Nos dados coletados ao final do semestre, os alunos do método de aprendizagem cooperativa foram comparados com alunos das demais turmas.

Bonaparte (1990) conduziu um estudo com 240 alunos do segundo ano do ensino fundamental. O estudo examinou a eficácia da aprendizagem colaborativa e métodos de aprendizagem competitivos em termos de desempenho matemático e auto-estima. Como resultado, ele descobriu que as aulas nas quais o método de aprendizagem cooperativa foi usado eram significativamente diferentes das aulas nas quais o método de aprendizagem competitiva era usado em termos de desempenho em matemática e autoestima.

Mark, Volk e Hinckley (1991) conduziram um estudo para investigar o impacto do regime de aprendizagem colaborativa na utilidade acadêmica de alunos que fizeram experiências no laboratório de química. No estudo, o estudo foi produzido pela criação de laboratórios entre os grupos controle e experimental. Ao final do estudo, concluiu-se que o grupo controle no qual foi utilizado o método de aprendizagem colaborativa foi mais bem-sucedido do que o grupo experimental no qual foram utilizados os procedimentos tradicionais.

George (1994) investigou o efeito de estratégias de aprendizagem colaborativa em salas de aula de universidades de diferentes culturas. na pesquisa comparou o método de aprendizagem tradicional com o método de aprendizagem por treinamento colaborativo. Os alunos que não tinham conhecimento prévio do estudo escolheram suas atribuições em sala de aula. Nos pré-testes aplicados antes do início do estudo, verificou-se que não houve diferença significativa na área disciplinar e nas atitudes de ambos os grupos, e nos pós-testes aplicados após o término do estudo, desenvolveu-se uma diferença significativa. em favor do experimental na medida em que o método de aprendizagem colaborativa poderia ser abordado.

Coppola e Lawton (1995), investigaram em um plano de atividades de aprendizagem colaborativa, orientadas para tornar o problema compreensível, identificar as lacunas e contribuir para o desenvolvimento, utilizar ferramentas e técnicas na resolução de problemas, suprir as necessidades das disciplinas quantitativas e qualitativas, e desenvolver estudos laboratoriais colaborativos. Ao final da pesquisa, concluíram que os alunos foram estimulados a desenvolver habilidades sociais e técnicas combinadas ao finalizar a aplicação do método de aprendizagem colaborativa com o currículo que desenvolveram.

Dougherty, et.al (1995) investigou o efeito do método de aprendizagem colaborativa no curso de química geral na melhoria do desempenho dos alunos, habilidades de retenção, 
atitudes e habilidades de comunicação. Na pesquisa, a universidade analisou o curso de Química Geral em três partes. Nestas partes, realizaram o seu trabalho com várias atividades como trabalhos de grupo formais, reuniões de grupo fora da aula, trabalhos de grupo, agrupamentos de grupo, resolução de questões de química por email e email. Como resultado do estudo, eles encontraram oposições características no desempenho do aluno, habilidades de retenção de memória, habilidades de comunicação e atitudes do grupo experimental ao qual o método de aprendizagem colaborativa foi aplicado,

Leung e Chung (1997) investigaram as consequências do método de treinamento colaborativo sobre o interesse e a moderação dos alunos na apresentação do ensino do professor e do curso de tecnologia educacional. Como resultado, eles descobriram que o método de aprendizagem colaborativa teve um efeito positivo sobre o desempenho acadêmico, o público de ensino e as condições de aprendizagem.

Nembhard (1997) investigou o efeito do método de aprendizagem colaborativa no ensino de simulação. A pesquisa foi conduzida com alunos do Departamento de Engenharia Industrial e de Sistemas da Auburun University. O pesquisador aplicou o método de aprendizagem colaborativa aos alunos do curso de simulação. Como resultado de suas observações e análises durante o curso, ele descobriu que os alunos desenvolveram habilidades de resolução de problemas e forte raciocínio sobre os conceitos.

Potthast (1999) teve como objetivo determinar os resultados da utilização do método de aprendizagem colaborativa em aulas introdutórias de estatística. Ele realizou o estudo em uma universidade na cidade oriental dos Estados Unidos. Foram avaliados 51 alunos que cursavam o primeiro ano desta universidade, trinta deles foram incluídos no estudo com o método de aprendizagem colaborativa e 21 deles foram incluídos no curso de estatística de estudos sociais com o método tradicional. Como resultado da avaliação dos quatro minitestes aplicados durante o estudo, o método de aprendizagem colaborativa, que é um dos dois métodos de ensino, afetou significativamente o desempenho acadêmico dos alunos do curso introdutório de estatística.

Ramsay, et.al. (2000) conduziram uma investigação sobre se as características cognitivas dos alunos são afetadas pela expertise do treinamento colaborativo (sobre o benefício do método de aprendizagem colaborativa na educação). Ao final do estudo, eles mostraram que as características cognitivas dos alunos estavam relacionadas a diferentes abordagens de aprendizagem. Concluíram também que o método de aprendizagem colaborativa pode ser mais eficaz nas características cognitivas dos alunos.

Haller, et.al (2000) investigou o nível acadêmico de estudantes de engenharia na North Carolina State University e o efeito da aprendizagem colaborativa na dinâmica da comunicação entre pares. A pesquisa comparou a eficácia do método de aprendizagem 
colaborativa e do método tradicional de aprendizagem em atividades educacionais. Diante dos dados obtidos ao final do estudo, concluiu-se que os alunos obtiveram grande sucesso nas turmas em que foi utilizado o método de treinamento colaborativo no ensino de engenharia, considerando os resultados positivos dos dados sobre confiança pessoal, atitude perante experiências educacionais, o desenvolvimento de habilidades dos alunos e disposição de aprendizagem, que foi detectado.

Quarstein e Peterson (2001) em cursos de política e estratégia de negócios investigaram os efeitos do método de aprendizagem colaborativa e do método de aprendizagem tradicional em alunos que perceberam o nível do curso. Como derivação da exploração, constatou-se que os alunos que utilizaram o método de aprendizagem colaborativa atingiram o nível necessário de habilidades de comunicação, habilidades e critérios conceituais de habilidades, sem ganho ou perda significativa.

Veenman, et.al (2002) investigou os efeitos complementares de um curso sobre aprendizagem colaborativa para futuros professores, o curso foi realizado em duas faculdades de formação de professores diferentes na Holanda. Um curso no método de aprendizagem colaborativa, como dependência positiva, interação face a face, habilidades sociais e operações em grupo com base nas primeiras e últimas observações do curso. Eles descobriram implicações comportamentais significativas para quatro dos cinco elementoschave essenciais para a atividade.

Brewer, Klein e Mann (2003) investigaram o efeito da aprendizagem colaborativa no desempenho, nas atitudes das disciplinas e nas habilidades de comunicação de adultos que abandonaram o ensino médio, mas desejam entrar e terminar o ensino médio novamente. No final do estudo, foi determinado que os alunos adultos que trabalham juntos em pequenos grupos desenvolveram atitudes muito mais elevadas em relação à área de assunto do que os trabalhadores individuais.

Box e Little (2003) investigaram o desempenho acadêmico e o desenvolvimento de conceitos pessoais de alunos do ensino fundamental com pequenos grupos na gestão da aprendizagem colaborativa. Ao avaliar os dados obtidos no final do estudo, as abordagens de pequenos grupos do Jigsaw mostraram ter vantagens e essas vantagens foram expressas em duas categorias: acadêmicas e psicológicas. A razão pela qual é academicamente vantajoso, foi determinado que o desenvolvimento do conceito pessoal dos alunos nos grupos experimentais também é positivamente eficaz. A vantagem psicológica é que alunos e professores fornecem mais motivação no método tradicional.

Stockdale e Williams (2004) investigaram o efeito do método de aprendizagem colaborativa no nível universitário em alunos de nível alto, médio e baixo em termos de desempenho nos testes. Como resultado, eles descobriram que os alunos com pontuações 
baixas e médias em comparação com os alunos que trabalharam de forma colaborativa aumentaram seu desempenho nos testes, mas os alunos com desempenho alto anteriormente tiveram uma diminuição insignificante em seu sucesso.

\section{Sites com uma abordagem de aprendizagem colaborativa}

Acredito que esses sites serão úteis para se chegar a informações e estudos relacionados ao método de aprendizagem colaborativa em um curto espaço de tempo. Os sites são ordenados com seus endereços da web:

- Site de Ted Panitz:aprendizagem colaborativa neste site, existem livros, artigos, pesquisas de professores, exemplos e outros links para sites sobre o método. home.capecod.net

- Aprendizado colaborativo: Colaboração da Engenharia nesta página web, há informações sobre ela, estudos realizados com videoteipe: clte.asu.edu

- Especialistas em educação e treinamento: Neste site, há gravações de vídeo de aprendizagem ativa, aprendizagem colaborativa, trabalho experimental, estilo educacional e de treinamento elaborado por um especialista: multimedia.vt.edu

- IASCE:Links estão incluídos neste site, estudos internacionais de educação colaborativa. Além disso, este site também inclui um mecanismo de busca para trabalho colaborativo: iasce.net

- SMET: Este site inclui atividades de treinamento em ciências, matemática, engenharia e tecnologia relacionadas à cooperação: wcer.wisc.edu

- Educação na web: sobre o método de colaboração nos recursos deste site, atividades do curso relacionadas a este método: bestpractice.net

- STEMMeta - Análise: neste site, os resultados acadêmicos dos alunos e há metaanálises sobre o método colaborativo de atitudes e atitudes: wcer.wisc.edu

- Trabalho em equipe: Trabalho em grupo, formação de grupo, apresentações orais e escritas em grupo, resultados de dados relacionados a estudos de método colaborativo estão incluídos neste site: ncsu.edu

- Centro de Métodos de Aprendizagem Colaborativa da Universidade de Minnesota: Este site inclui como o método de aprendizagem cooperativa é aplicado, quem está trabalhando neste método e os resultados do estudo sobre o método: clcrc.com 


\section{Conclusão}

Dependendo das inovações científicas e tecnológicas das sociedades contemporâneas, a cooperação entre grupos e dentro deles tornou-se muito importante. A importância disso pode ser facilmente determinada por indivíduos na sociedade. Nos últimos anos, qualquer gerente de empresa ou diretor de escola percebeu a necessidade de cooperar efetivamente com administradores e funcionários. Os esforços para administrar uma escola ou empresa sem cooperação estão se tornando impossíveis a cada dia.

Esta situação é válida hoje em muitos campos empresariais e profissionais, mas também em novas intervenções mútuas. Em perspectiva, pode-se perceber facilmente que as relações sociais do ser humano, que é um ser social, devem se desenvolver. Como se sabe, o objetivo da escola não é apenas dar aos alunos determinadas aulas e informá-los sobre os assuntos que desejam conhecer, mas também contribuir para o seu desenvolvimento físico, mental, emocional e social.

A necessidade de determinar os métodos mais adequados e trabalhar de acordo com esses métodos para cumprir essas obrigações no processo de ensino-educação é um tema com o qual todos os professores estão familiarizados. Nesse contexto, sabe-se que o procedimento de treinamento cooperativo afeta positivamente o desenvolvimento dos indivíduos em vários aspectos, bem como em sua aprendizagem.

O método de aprendizagem colaborativa auxilia os alunos a adquirirem a habilidade de trabalhar em equipe, que se expressa como o segredo do sucesso. Neste estudo, o método de aprendizagem colaborativa foi examinado. Embora os alunos utilizem diferentes ferramentas (projetos, situações-problema, questões de pensamento crítico) para alcançar os objetivos deste método de aprendizagem, o objetivo principal deste método é: acesso a recursos de informação; é formar indivíduos que dêem importância às ideias dos outros e ao mesmo tempo lhes dê sentido, que questionem, que possam se comunicar de maneira eficaz com o meio ambiente, ou seja, que aprenderam a aprender.

No mundo de hoje, a enxurrada de informações e a rápida distribuição de informações tornaram o modelo humano de acumulação de conhecimento inaceitável. Em vez de, há necessidade de um modelo humano que possa questionar, pensar, discutir, mudar, resolver problemas e liderar. Esta é uma das razões pelas quais o método de aprendizagem colaborativa é cada vez mais usado.

Os principais motivos que tornam o método de aprendizagem colaborativa atraente podem ser listados abaixo:

- Filosofia educacional: é um método de aprendizagem ativo 
- Propósito: Permita que os alunos aprendam cooperativamente.

- Ponto de partida: Processo de conteúdo do tópico

- Os alunos trabalham em grupos:para entender o conteúdo do assunto. eles trabalham.

- Aluna:Concorrente. você e os membros do seu grupo. é responsável pela aprendizagem.

- Professora:Ele é um guia e facilitador. Crie grupos. A aprendizagem estabelece metas.

- Medição e avaliação: Medição de alternativas que utilizam técnicas de avaliação. 


\section{Referências}

Acikgoz, KU, (1992). Aprendizagem cooperativa: teoria, pesquisa, prática. Escritório de impressão, Malásia.

Acikgoz, KU, (2003). Active Learning, Education World Publications, Print.

Bonaparte, EPC, (1990). O efeito da organização cooperativa da sala de aula para o domínio da aprendizagem no desempenho matemático e na autoestima de alunos urbanos do segundo ano. resumo da dissertação internacional de resumos.

Box, JA, Little, DC, (2003). Instrução cooperativa em pequenos grupos combinada com organizadores avançados e sua relação com o autoconceito e a realização de estudos sociais dos alunos do ensino fundamental. Journal of Instructional Psychology.

Brewer, AS, Klein, DJ e Mann, KE, (2003). Uso de estratégias de aprendizagem em pequenos grupos com alunos adultos de reentrada, College Student Journal.

Buyukkaragoz, S. e Civi, C., (1997). Métodos gerais de ensino, educação especial.

Cooper, J., Mueck, R., (1990). Participação do Aluno na Aprendizagem: Aprendizagem Cooperativa e Instrução Universitária, Jornal de Excelência no Ensino Universitário.

Coppola, BP e Lawton, RG, (1995). Quem tem a substância que eu tenho? Um plano para atividades de aprendizagem colaborativa. Journal of Chemistry Education.

Davis, BG, (1993). Aprendizagem colaborativa: trabalho em grupo e equipes de estudo, ferramentas para o ensino, San Francisco.

Delen, H., (1998). Cooperativa no curso de estudos sociais do quinto ano do ensino fundamental $\mathrm{O}$ efeito do método de aprendizagem no desempenho acadêmico, Dissertação de Mestrado, Adana

Demirel, O. ve Un, K., 1987. Educational Terms, Dawn Press.

Doolittle P., (1997). Zona de desenvolvimento próximo de Vygotsky como uma fundação para aprendizagem cooperativa. Revista de excelência no ensino universitário.

Dougherty, RC, Bowen, CW, Berger, T., Rees, W., Mellon, EK, Pulliam, E., (1995). Aprendizagem cooperativa e comunicação aprimorada, Journal of Chemistry Education.

Doymus, K., Bayrakceken, S. e Simsek, U., 2003. Preparando o efeito da tarefa colaborativa no sucesso, Journal of the Kazım Karabekir Faculty of Education.

Doymus, K., Simsek, U. e Bayrakçeken, S., 2004. Método de aprendizagem cooperativa O efeito do sucesso acadêmico e atitude na aula de ciências, Turkish Journal of Science Education.

Frierson, H., (1986). Dois métodos de intervenção: efeitos em grupos de notas do conselho de enfermagem de estudantes predominantemente negros, Journal of Research and Development in Education.

Gardener, BS e Korth, SD, (1996). Uso de reflexão em grupos de aprendizagem cooperativa para integrar teoria e prática. Revista de excelência no ensino universitário.

George, PG, (1994). O efeito das estratégias de aprendizagem cooperativa em salas de aula universitárias multiculturais. Revista de excelência no ensino universitário. 
Haller, RC, Gallagher, VJ, Weldon, TL, Felder, RM, (2000). Dinâmica das interações entre pares na aprendizagem cooperativa, Diários de Educação em Engenharia.

Johnson, DW, Marumay, G., Johnson, RT, Nelson, D. e Skon, L., (1981). Efeito das estruturas de metas cooperativas, competitivas e individualistas na realização: uma meta-análise. Boletim psicológico.

Johnson, DW, Johnson, RT, Holubec, EJ e Roy, P., (1984). Círculos de Aprendizagem: Cooperação na Sala de Aula, Alexandria, VA: Associação para Monitoramento e Desenvolvimento Curricular, Minnesota.

Johnson, DW e Johnson, RT, (1988). Círculos de aprendizagem: cooperação em sala de aula, Estados Unidos.

Johnson, DW e Johnson, RT, (1992). Abordagens para a implementação da aprendizagem cooperativa na sala de aula de estudos sociais, Aprendizagem cooperativa na sala de aula de estudos sociais: um convite ao estudo social, RJ, Stahl e RL, Vansicle Editor: Washington National Council for Social Studies. Boletim No: 87.

Leung, CD e Chung, C., (1997). Desempenho do aluno em um curso de tecnologia de ambiente educacional aprimorado pelo aprendizado cooperativo, Journal of Science Education and Technology.

Mallinger, M., (1998). Aprendizagem Colaborativa Transfronteiriça: Lidando com a Resistência dos Alunos, Revista sobre Excelência no Ensino Universitário.

Mark, ES, Volk, GL e Hinckley, CC, (1991). Aprendizagem cooperativa no laboratório de graduação, Journal of Chemical Education.

Milis, B., (1991). Cumprindo totalmente a promessa dos sete princípios por meio da aprendizagem cooperativa: Uma agenda de ação para a sala de aula universitária, Jornal de Excelência em Ensino Universitário.

Nembhard, HB, (1997). Cooperative Learning in Simulation, Proceedings of the 1997 Winter Simulation Conference.

Potthast, MJ, (1999). Resultados do uso de aprendizagem cooperativa em experiências de pequenos grupos em cursos introdutórios à estatística, diário do estudante universitário.

Quarsitein, VA, Peterson, PA, (2001). Avaliação da aprendizagem cooperativa: uma abordagem baseada em objetivos, ensino superior inovador.

Ramsay, A., Hanlon, D. e Smith, D., (2000). A associação entre o estilo cognitivo e a preferência dos alunos de contabilidade pela aprendizagem cooperativa: uma investigação empírica, Journal of Accounting Education.

Simsek, U., Saturated, K., Simsek, U., e Ozdemir, Y., 2004. Students Investigation of the effect of group learning method on democrático atitudes, Journal of Kazim Faculty of Education.

Simsek, U., Doymus, K. e Kızıloglu N., 2005, Conhecimento de Alunos do Ensino Médio e Habilidades Adquiridas pelo Método de Aprendizagem em Grupo, Kastamonu Journal of Education. 
Simsek, U., 2005, Método de Aprendizagem Cooperativa Curso de Ciências Acadêmicas Effect on Success and Attitude, Dissertação de Mestrado, Erzurum.

Slavin, RE, (1988). Métodos de Grupos Pequenos, Enciclopédia Internacional sobre Ensino de Educação e Treinamento de Professores, Oxford: Pergamon University Press

Slavin, RE, (1990). Métodos Abrangentes de Aprendizagem Cooperativa: Incorporando a Aprendizagem Cooperativa no Currículo e na Escola, Aprendizagem Cooperativa: Teoria e Pesquisa Slavin, RE, 1990, (editor: Shlomo Sahran), Nova York

Stockdale, SL, Williams, RL, (2004). Grupos de aprendizagem cooperativa de nível universitário: efeitos diferenciais nos alunos com exames de alto, médio e baixo rendimento. Journal of Behavioral Education.

Strohl, M. e Schneck, S., (1991). Native American Cooperative Learning Activities, Scholastic's Professional Books, Impresso nos EUA

Susan, SE e Susan, FW, 1990. Livro de Ensino: Estratégias Cooperativas Aprendendo e Iniciando a Vida Escolar Profissional, Impresso nos EUA.

Topsakal, S., (1999). Educação científica, Editorial Alfa Distribución. Istambul.

Treisman, U., (1985). Um Estudo do Desempenho Matemático de Estudantes Negros na University of California, Berkley (Ph.D. Thesis, University of California, Berkley, 1986), Dissertation Abstracts International.

Veenman, 6S4.1, Benthum, N. van, Bootsma, D., Dieren, J.van, Kemp, N. van der, (2002). Aprendizagem cooperativa e formação de professores, ensino e formação de professores.

Watson, SB (1992). Os elementos essenciais da aprendizagem cooperativa, o americano, professor de biologia.

Webb, NM, (1985). Interação e aprendizagem do aluno em pequenos grupos, Learning to Cooperate, Cooperate to Learn, (Editado por Robert Slavin et al.), New York Press Plenum.

Webb, NM, Sydney, H. e Farivor, AM, (2002). Teoria na Prática, Universidade de Educação. Young, C. e Young, L., (1999). Avaliação da aprendizagem em cursos interativos, Revista de excelência no ensino universitário. 